\title{
Regular rinsing with chlorhexidine does not reduce caries in older adults
}

\author{
Abstracted from \\ Wyatt CC, Maupome G, Hujoel PP, et al. \\ Chlorhexidine and preservation of sound tooth structure in older adults. A placebo-controlled trial. \\ Caries Res 2007; 41: 93-101 \\ Address for correspondence: Dr Chris CL Wyatt, Department of Oral Health Sciences, \\ Faculty of Dentistry, University of British Columbia, 2199 Wesbrook Mall, Vancouver, \\ British Columbia V6T 1Z3, Canada. E-mail: cwyatt@interchange.ubc.ca
}

\section{Question: Is a daily chlorhexidine mouthrinse effective at reducing caries in a community- dwelling elderly population?}

Design This was a placebo-controlled randomised clinical trial (RCT). Intervention An active daily rinse of $0.12 \%$ chlorhexidine (CHX) was compared with a similar placebo rinse. Subjects alternated between daily rinsing for 1 month, followed by weekly rinsing for 5 months for 5 years

Outcome measure The primary outcome was the probability of transition from a sound to a nonsound tooth (an 'event') in each group, specifically, a transition from a sound surface to a surface that became extracted, filled or decayed. Two secondary analyses were performed: an analysis where surfaces that were extracted without an intervening state of decayed or filled were censored, and an analysis where surfaces that were extracted or filled without ever being called decayed at any time were censored. The primary test statistics for evaluating treatment effects was based on the proportional hazards model.

Results Over the 5-year course of the study, 273 elders (24.8\%) did not complete all five followup visits; 57 (5.2\%) died, and 15 (1.4\%) became edentate. This was lower than the $10 \%$ attrition rate every year that had been projected at baseline. The study failed to identify an effect of regular $\mathrm{CHX}$ rinsing on the preservation of sound tooth structure on either the roots or the crowns of teeth in low-income older adults. Caries attack rates and hazard ratios are shown in Tables 1 and 2. No adverse events, including anaphylactic reactions, rashes or gastrointestinal disturbances, were reported by the subjects.

\section{Table 1. Caries attack rates}

\begin{tabular}{l|c|c} 
& \multicolumn{2}{|c}{ Caries attack rate* $\mathbf{( 9 5 \% ~ C l )}$} \\
\hline Surface & Chlorhexidine group & Placebo group \\
\hline Coronal & $1.98(1.72-2.29)$ & $2.24(1.86-2.54)$ \\
\hline Root & $3.01(2.72-3.61)$ & $3.28(2.78-3.86)$
\end{tabular}

*Number of events per 100 surfaces

$\mathrm{CI}$, Confidence interval.

\begin{tabular}{|c|c|c|c|}
\hline Event & HR & $95 \% \mathrm{Cl}$ & $\mathbf{P}$ \\
\hline \multicolumn{4}{|l|}{ Transition from sound surface to: } \\
\hline $\begin{array}{l}\text { restored, decayed or extracted } \\
\text { (coronal surfaces) }\end{array}$ & 0.88 & $0.71-1.08$ & 0.21 \\
\hline $\begin{array}{l}\text { restored, decayed or extracted (root } \\
\text { surfaces) }\end{array}$ & 0.92 & $0.74-1.14$ & 0.42 \\
\hline $\begin{array}{l}\text { restored or decayed (coronal } \\
\text { surfaces) extracted surfaces censored }\end{array}$ & 0.85 & $0.70-1.03$ & 0.11 \\
\hline $\begin{array}{l}\text { restored or decayed (root surfaces) } \\
\text { extracted surfaces censored }\end{array}$ & 0.96 & $0.78-1.18$ & 0.68 \\
\hline $\begin{array}{l}\text { decayed (coronal surfaces) extracted } \\
\text { and filled surfaces censored }\end{array}$ & 0.92 & $0.69-1.23$ & 0.59 \\
\hline $\begin{array}{l}\text { decayed (root surfaces) extracted } \\
\text { and filled surfaces censored }\end{array}$ & 0.97 & $0.70-1.33$ & 0.83 \\
\hline
\end{tabular}

Conclusions These findings suggest that regular rinsing with $\mathrm{CHX}$ does not have a substantial effect on the preservation of sound tooth structure in older adults.

\section{Commentary}

This RCT aimed to assess the effectiveness of CHX mouthw ash at reducing coronal and root caries. The population group participating in the trial comprised people of age 60-75 years. Although this group lived in the community rather than in an institution, they had a history of limited access to dental services and poor daily oral healthcare. It was felt important to use this age group because previous studies have focussed on the use of CHX in children, and so the results may not be transferable.

This is an increasingly relevant topic in dental public health, given the changing demographics of our population, with a rising number of older people, many of whom are retaining more natural teeth into older age. Prevention of coronal and root caries is becoming ever more important for this section of the population.

This study's results failed to show that regular use of CHX had any effect on the preservation of tooth structure, compared with placebo. There seems to be some doubt, however, over the inactive nature of the placebo, as it contained quinidine, which ironically may itself have had some therapeutic effect! This anomaly should certainly be addressed in any future studies of this nature. 


\section{CARIES}

Other problems with the analysis relate to the differences in undertaking studies with older people rather than children, eg, when following up teeth that have been extracted: there are many more reasons for tooth loss in adults than children. Further work is also required to develop more appropriate clinical caries measures for this age group, perhaps at the tooth level rather than surface level, because of the heavily restored dentition.

For the individual patient, there may still be some merit in recommending CHX for particular oral conditions, but in terms of public health interventions, it seems better to concentrate preventive efforts on the promotion of fluorides. Another study by authors from the same group showed the use of $0.2 \%$ sodium fluoride mouthwash on a daily basis for 2 years to be significantly more effective than $\mathrm{CHX}$ in reducing the incidence of caries, particularly root caries. ${ }^{1}$
There has also been a recent systematic review of the use of fluorides for the prevention of root caries in older adults, ${ }^{2}$ which showed that high fluoride toothpaste (5000 ppm) and daily fluoride mouthwash $(0.05 \%)$ both had a beneficial effect on the reduction of root caries.

\section{Maura Edwards}

Department of Public Health, National Health Service Ayrshire and Arran, Ayr, Scotland, UK

1. Wyatt CCL, MacEntee MI. Caries management for institutionalized elders using fluoride and chlorhexidine mouthrinses. Community Dent Oral Epidemiol 2004; 32 322-328.

2. Heijnsbroek M, Paraskevas S, Van der Weijden GA. Fluoride interventions for root caries: a review. Oral Health Prev Dent 2007; 5: 145-152.

Evidence-Based Dentistry (2009) 10, 13-14. doi:10.1038/sj.ebd.6400628 